\title{
Effects of HoMnO3 nanoparticles addition on microstructural, superconducting and dielectric properties of $\mathrm{YBa} 2 \mathrm{Cu} 3 \mathrm{O} 7-\delta$
}

\begin{abstract}
$(\mathrm{YBa} 2 \mathrm{Cu} 3 \mathrm{O} 7-\delta) 1-x(\mathrm{HoMnO} 3) x(x=0.0,0.0025,0.005,0.01,0.03$ and 0.05$)$ ceramics were fabricated by introducing co-precipitation synthesized HoMnO3 (HMO) nanoparticles during solid state reaction process of $\mathrm{YBa} 2 \mathrm{Cu} 3 \mathrm{O} 7-\delta$ (Y-123) superconductors. (Y-123)1- $x(\mathrm{HMO}) x$ samples were characterized using X-ray diffraction (XRD), field emission scanning electron microscope (FESEM) attached with energy Dispersive X-ray spectrometer (EDX), four-point probe measurement, AC susceptometer and impedance analyzer. Majority of XRD patterns were indexed to orthorhombic Y-123 phase. Besides, YBaMn2O5 (1.5-3.6\%) and YBaMn2O6 (2.4-7.4\%) phases were detected. FESEM images and EDX analysis showed the presence of agglomerated particulates related to $\mathrm{Mn}$ and Ho based phases residing in between the Y-123 grains. The superconducting behavior was significantly enhanced at $x=0.0025$ while there was no major depression noticed in critical temperature $(T \mathrm{c}-\mathrm{R}=0)$ as the addition increased till $x=0.03(T \mathrm{c}-\mathrm{R}=0=88 \mathrm{~K})$. AC susceptibility curves of composites samples manifested sharp transitions for samples with $x=0.0025$ and 0.005 . Dielectric parameters $\varepsilon \mathrm{r}^{\prime}$ and $\varepsilon \mathrm{r}^{\prime \prime}$ decreased as the frequency increased for all samples. The $\varepsilon \mathrm{r}$ versus frequency measurements showed increment in $\varepsilon \mathrm{r}^{\prime}$ and $\varepsilon \mathrm{r}^{\prime \prime}$ values for all added samples as compared to Y-123 sample. The highest values for $\varepsilon \mathrm{r}^{\prime}$ and $\varepsilon \mathrm{r}^{\prime \prime}$ were obtained for sample $x=0.05$ with the highest loss at lower frequency. The Nyquist plots of complex impedance were analyzed where two semi-arc circulars represent grain and grain boundary effect were deduced.
\end{abstract}

Keyword: Y-123; HoMnO3; Critical temperature; Solid state reaction; Co-precipitation; Impedance 
